\title{
A Key-Ordered Decisional Learning Parity with Noise (DLPN) Scheme for Public Key Encryption Scheme in Cloud Computing
}

\author{
Tarasvi Lakum ${ }^{1}$ \\ Department of CSE \\ Koneru Lakshmaiah Education Foundation \\ Vaddeswaram, A.P, INDIA
}

\author{
B.Thirumala Rao*,2 \\ Department of CSE \\ Koneru Lakshmaiah Education Foundation \\ Vaddeswaram, A.P, INDIA
}

\begin{abstract}
The variation of decisional learning parity with noise (DLPN) named as key-Ordered DLPN based security algorithm is presented in this work. The proposed scheme uses DLPN by extending it to an even-odd-order scheme, depend on the value of probability distribution of odd and even bits for encryption, where odd and even bits are the input integer values for key generation algorithm. This states that the probability distribution of odd and even bits are ordered based on the key generation, the process of odd and even bits resolving is the solution of DLPN attacker problems, thus, the proposed scheme provides more correctness and security proof. Through the learning parity with noise (LPN), DLPN and RSA algorithms, the proposed system is evaluated, to measure the encryption time, public key and ciphertext bits.
\end{abstract}

Keywords-LPN; DLPN; RSA; key-ordered; time

\section{INTRODUCTION}

To provide data security, in a confidential and in an authorized encryption, there is a need of protection to the information from an unauthenticated user [1-3]. The information required should be made available to the authorized users and to be protected from unauthorized users by creating it unavailable. Through which the availability, confidentiality and integrity of data become necessary for the security of data.

Recently, an encrypted security [4] [20-21] became an ideological research area, with a process of keeping the data in a server and encrypted form of data is communicated, in a way for the purposed users can have access and process. Cryptography broadly made in [1], [5-8] to a symmetric and a public-key. In asymmetric cryptography, public key is used for encryption process and private key is used for decryption process. The prior is more important and secure than the later for cryptography, which depends on the length of the key used [4-9] and work of cryptography made during the computations.

From the survey works, motivated with the challenges in cryptography, a variation of DLPN with two order bits has been proposed, where the keys are dependent of LPN variables and is possible to enhance the scheme by odd and even bits with newly computed bits during the process of encryption and decryption [9-15]. Increased key generation time can be reduced by increasing the process of coding, it made a big-task in the implemented method, to provide security by the process, from attacks and made secure [16-28].

\section{A. $L P N$}

LPN [19] computational version is an analogue of linear codes decoding through random numbers, which is an NPcomplete problem. The improvement in the efficiency is made through the sparse Fourier spectrum, that is LPN solver through $2^{O(n / \log n)}$ constant term with $\mu=O(1)$, is $\leq 0.5 \&$ independent of secret size for all the values of $n$, is represented as n. Here $q=\operatorname{poly}(n)$ is the number of training samples, with this the time complexity reaches to the training samples, for $q=O(n)$, and for $q=n+O(1)$ the time complexity goes to $2^{O\left(n^{1-e}\right)}$; based on these, is the main drawback of the LPN, has to be reduced.

\section{B. $D L P N$}

In this, the public key becomes small, having a random public and private key vectors $a \in Z_{2}^{n}$ and $s \in Z_{2}^{n}$ respectively, based on these if an attacker gets $(a,\langle a, s\rangle+e)$, where $e \leftarrow \mathrm{Ber}_{\tau}$, occurring only between $0<\tau<1$. But from the noise rate of LPN the distribution is $0<\tau<0.5$, through which attacker is able to differentiate the random $r \leftarrow Z_{2}$ and sampling $\langle a, r\rangle$ elements, which should be solved through DLPN [29], which is a public-key encryption scheme to improve the security.

A DLPN attacker with $(a,\langle a, s\rangle+e)$ set, where $e \leftarrow$ $\operatorname{Ber}^{n x n}{ }_{\tau}$, remaining parameters at DLPN based. To distinguish between the random $r \leftarrow Z^{n x n}$ and sampling $\langle a, r\rangle$ elements, with a new sample $(a,\langle a, s\rangle+e)$, taking $\tau=O(1 / \sqrt{n})$ noise rate and randomly selected public and private key vectors $a \in Z_{2}^{n x n}$ and $s \in Z_{2}^{n x n}$ respectively, by assuming the DLPN probabilistic polynomial time (PPT) parameters $(n, \tau)$ is negligible.

\section{Contributions}

The proposed work in this paper is an approach through the tradition public key cryptography scheme RSA [26-28] and current public key cryptography schemes LPN [29] and DLPN [29], so we restrict our discussions and contributions among RSA, LPN and DLPN only. This paper provides new constructions of encryption schemes from a variant of DLPN.

*Corresponding Authors 
First contribution is to introduce a DLPN variety problem with $S \leftarrow \operatorname{Ber}^{n x n}{ }_{\tau}$ within the assumptions of normal DLPN problem. As a second contribution a key-bit is constructed into vector-bit through cryptographic operations. During the cryptographic decryption process, the $\mathrm{n}$ dimensional vector is having the hamming weight of $\mathrm{n} / 2$, with the plaintext-bit as even or odd or vice-versa. The probability order of odd and even plaintext-bits is monitored to decay their exponential exceeding expectations, through by reducing the error probability. In the third contribution, the odd and even plaintext-bits are ordered in a multi-bit level based on the encryption and decryption algorithm of the public key. Unlike the previous schemes, the proposed scheme is a minimization to the LPN and DLPN problem. Proposed authentication scheme is efficient as the surveyed schemes.

\section{RELATED WORKS}

\section{A. Algorithms}

Many schemes have been proposed for public key cryptography. In this paper, the contributions are on RSA algorithm, LPN and DLPN.

In RSA algorithm [22-25], it becomes difficult to find the decryption key under the large integer's factors. An enhanced RSA algorithm is proposed by factorizing and deriving the key variable and considering the third prime number by making the complexity more and robust. A new factor should be replaced to increase the complexity at cryptography process to reduce the track back difficulty in the product of three prime numbers, by achieving the increased time complexity.

In LPN [19],[29], the problems available are made in to two non-trivial solving methods, one is a type of method which intends for all possible noise vectors to be intended and the other which has a sub index time complexity $2^{O(n / \log n)}$. This complexity is increased further in to $2^{O(n / \log \log n)}$ with the sampling time of $n^{1+\varepsilon}$. A further improvement in the algorithm with less running time is to be made, and there is a need of polynomial time algorithms to solve the variety of LPN problems. So there is a need of a design for LPN based cryptographic applications, through symmetric encryption in public key scheme. Here a LPN based on public key encryption with the noise ratio of $\tau \approx 1 / \sqrt{n}$ is considered. However, in all the variants of, an encoding error prevails which is a nonnegligible. To solve these, in this paper, a matrix LPN problem is considered to solve the encoding error problem through Damgård's scheme.

In DLPN [29], the problem is to vary between the uniform distribution over the $\mathrm{Z}$ value and the number of samples given by the oracle LPN. It can be formulated by an optimization solution i.e., by using random matrix A with a random column vector $\mathrm{c}$ over $\mathrm{Z}$, to find the vector $\mathrm{v}$ to maximise the equations of the scheme is $A v=c$. This illustrates a problem of decrypting a NP-hard, which is a random linear code. To solve these variants of LPN problems, require a sub-exponential query during the sub-exponential time. The DLPN is a variant of $\mathrm{LPN}_{1}$ problem, with a distributed secret $\mathrm{s}$ is a uniform random variable and through $\mathrm{Ber}_{\tau}^{k}$. Here noise parameter made nonconstant and it depends on the value of $\mathrm{k}$, through a linear number of queries which are arbitrarily polynomial and matrix version of $\mathrm{LPN}_{1}$. A public key cryptosystem based on $\mathrm{LPN}_{1}$ is given as $\left|\operatorname{Pr}_{s, A, e}[D(A, A \cdot s \Theta e)=1-\operatorname{Pr}[D(A, r)=1]]\right| \geq \epsilon$, where $A \leftarrow B_{\mu}^{q \times n}, s \leftarrow Z_{2}^{n}, e \leftarrow B_{\mu}^{q}$ and $U_{q}$ is a uniform distribution over $Z_{2}^{q}$. The $L P N_{n, \mu, n+q}$ problem is hard and makes the problem of Knapsack-LPN $N_{n, \mu, n+q}$ problem becomes hard. The DLPN problem is hard compare to LPN problem defined above, which leads to more complex results in public encryption key schemes, to make it available the design is made in black-box manner from the available DLPN problem identified, which is made for noise of $\mu=\omega(1) / \sqrt{ } n$.

\section{B. Mathematical Explanation}

1) LPN: To make PKE correct, the PKE should be PKE= (KeyGen,Enc,Dec) for all the messages $\mathrm{m} \in \mathrm{M}$, the equation is given by: $\quad \operatorname{Pr}\left[\operatorname{Dec}_{s k}\left(E n c_{p k}(m)\right) \neq m \mid(p k, s k) \leftarrow\right.$ $\left.\operatorname{KeyGen}\left(1^{z}\right)\right] \leq \operatorname{negl}(z)$, where $\operatorname{negl}(z)$ is negligible function. To discuss the LPN problem scenario, let us look in to the LPN oracle which is given by: $\left\{(v, b) \mid v \leftarrow Z_{2}^{k}, b=<\right.$ $\left.v, s>\oplus \epsilon, \epsilon \leftarrow B e r_{\tau 0}\right\} \in Z_{2}^{k+1}$, where $\left.s \leftarrow Z_{2}^{k}, \tau \in\right] 0, \frac{1}{2}[$ is a constant noise parameter and $B e r_{\tau}$ is the Bernoulli distribution with $\tau$ parameter. For the LPN search problem, depending on the distribution of As, $\tau$ the LPN oracle has the output independent random samples of $A_{s, \tau}^{L P N}$. Consider $L P N_{k, \tau}$ as an instance of LPN with a secret key of size $\mathrm{k}$ and the noise parameter as $\tau$. The algorithm which solves the $M(q, t, m, \theta)$ LPN search problem if $\operatorname{Pr}\left[M^{A_{s, \tau}^{L P N}}\left(1^{k}\right)=s \mid s \leftarrow Z_{2}^{k}\right] \geq \theta$. It distinguishes between the distribution over the $Z_{2}^{k+1}$ and the samples given by an LPN oracle. The search LPN problem is formulated as a optimization problem, through a random matrix $\mathrm{A}$, a random column matrix $\mathrm{b}$ over $\mathrm{Z}_{2}$, to find the vector $s$ which maximises the number of equations of the system As=b.

2) DLPN: The decisional LPN problem is defined by the parameters $n \leftarrow N, \tau \in R, \tau=\Theta(1 / \sqrt{n})$ and randomly selected matrix $A \leftarrow Z_{2}^{n \times n}, S \leftarrow Z_{2}^{n \times n}$ as random selected matrix. The sample set of key can be obtained by the attacker in the form of $(\mathrm{A}, \mathrm{AS}+\mathrm{E})$ by $E \leftarrow B e r_{\tau}^{n \times n}$, with the database sample (A,R), $R \leftarrow Z_{2}^{n \times n}$ having a non-negligible probability after getting the enough sample sets, which makes the DLPN problem to be solved. With the noise rate $\tau$, the DLPN assumptions are defined with the probabilistic polynomial time (PPT) attacker including the parameter $\mathrm{n}$ which is negligible and is defined as $\tau=\theta(1 / \sqrt{n})$. The bit level encryption of DLPN is:

a) Choose $\quad A \leftarrow Z_{2}^{n \times n}, S \leftarrow B e r_{2}^{n \times n}, E \leftarrow B e r_{\tau}^{n \times n} \quad$, compute $B=A S+E$ and $\operatorname{KeyGen}\left(1^{n}, \tau\right)$ and returns a public key $p k=(A, B)$ and a private key sk $=(S)$.

b) Choose encryption $\operatorname{Enc}(p k, m)$, public key pk and user message $m \in Z_{2}$, compute $c_{1}=r^{T} A+e_{1}^{T}, c_{2}=r^{T} B+$ $e_{2}^{T}+m I$ and returns to a ciphertext $c=\left(c_{1}, c_{2}\right)$.

c) Choose decryption $\operatorname{Dec}(s k, c)$, the private key sk and a ciphertext $c=\left(c_{1}, c_{2}\right)$, compute $d=c_{1} \times S+c_{2}$ and returns $\mathrm{m}=0$ for $h(d) \ll n / 2$, and $\mathrm{m}=1$ for $h(d) \gg n / 2$.

To provide correctness of DLPN problem, define $\mathrm{X} \sim \operatorname{Bin}_{\mathrm{n}, \tau}$ with an even variable with variations of $\frac{1}{2}+\left(1-2 \tau^{2}\right)^{2} / 2$, and 
define hamming weight of each column of odd variable with variations of $n\left(1-\left(1-2 \tau^{2}\right)^{n} / 2\right)$, with the selected scheme of $h(d) \ll n / 2$ to meet its reduced decryption error.

\section{PROPOSED Key ENCRYPTION SCHEME}

\section{A. Proposed Scheme}

Proposed scheme uses two prime messages in an order with the increased size. The message noise of these two orders generate the public key $(\mathrm{P})$, a variable $(\mathrm{O})$ and private key $(\mathrm{Q})$. $\mathrm{P}$ and $\mathrm{Q}$ are generated with $\mathrm{O}$ parameter considered. Random messages Even() and $\operatorname{Odd}()$ are required to create the prime messages. It takes a time in generation of secure key using Even() and $\operatorname{Odd}()$ messages and find the noise in the message and time taken is also less by dividing them in to two categories, which makes the reduction in complexity of the algorithm. The value of $\mathrm{O}$ is generated in a random way and these values are transmitted through a sequence of newly generated that is, O_EO as a public key. It continues with the $\mathrm{P}$ and $\mathrm{Q}$ through the regenerative $\mathrm{O}$ and $\mathrm{O} \_\mathrm{EO}$ sequences, so it becomes difficult to the attackers to enter the system which is encrypted, which helps system to improve the security. Proposed key encryption scheme algorithm is described as follows.

\section{B. Mathematical Representation of Proposed DLPN with two order bits Algorithm}

It includes:

1) kO-DLPN of kO_KeyGen $\left(1^{\mathrm{n}}, \tau\right)$ takes $\mathrm{n}$ as integer and $\tau$ as noise rate, by choosing a random matrix $A \leftarrow Z_{2}^{n \times n}, S \leftarrow$ $\operatorname{Ber}_{\tau}^{n \times n}, E \leftarrow \operatorname{Ber}_{\tau}^{n \times n}$.

2) $\mathrm{kO}$-DLPN of $\mathrm{kO} \_\operatorname{Enc}(p k, m)$ is divided in to two parts.

First is Even(), where $\mathrm{m}$ is converted to an even-square matrix $M^{\text {Even }} \in Z_{2}^{n \times n}$, if $\mathrm{m}_{\mathrm{e}}=1$, for $M^{\text {Even }} \mathrm{e}$-th column of is 1 and similarly at each entry of the e-th column is 0 , e.g., $\mathrm{m}=(0,1,1,1)^{\mathrm{T}}$, then $M^{\text {Even }}=\left[\begin{array}{llll}0 & 1 & 1 & 1 \\ 1 & 0 & 1 & 1 \\ 1 & 1 & 0 & 1 \\ 1 & 1 & 1 & 0\end{array}\right]$, by choosing $E^{\text {Even }} \leftarrow \operatorname{Ber}_{\tau}^{n \times n}$.

And second is $\operatorname{Odd}()$, where $\mathrm{m}$ is converted to a odd-square matrix $M^{\text {Odd }} \in Z_{2}^{\overline{n \times n}}$, if $\mathrm{m}_{\mathrm{e}}=0$, each entry of the e-th column of $M^{O d d}$ is 0 and similarly at each entry of the e-th column is 1 , e.g., $\mathrm{m}=(1,0,0,0)^{\mathrm{T}}$, then $M^{\text {Odd }}=\left[\begin{array}{llll}1 & 0 & 0 & 0 \\ 0 & 1 & 0 & 0 \\ 0 & 0 & 1 & 0 \\ 0 & 0 & 0 & 1\end{array}\right]$, by choosing $E^{O d d} \leftarrow B e r_{\tau}^{\overline{n \times n}}$.

3) kO-DLPN of kO_Dec( $s k, c)$ with ciphertext $C=$ $\left(C_{\text {Even }} \oplus C_{1}, C_{2} \oplus C_{\text {Odd }}\right)$.

C. Solution Equations for Proposed DLPN with two order bits Algorithm

It includes:

1) $\mathrm{kO}$-DLPN of $\mathrm{kO} \_\operatorname{KeyGen}\left(1^{\mathrm{n}}, \tau\right)$ : Compute $B=A S+$ $E$, to return $p k$ with key $s k=(S)$.

2) $\mathrm{kO}$-DLPN of $\mathrm{kO} \_$Enc $(p k, m)$ is divided in to two parts.
First is Even( $)$, returns to ciphertext $C=\left(C_{\text {Even }}\right.$ and $\left.C_{2}\right)$ where the computations are $\mathrm{C}_{\text {Even }}=\mathrm{RA}+\mathrm{E}_{\text {Even }}$ and $\mathrm{C}_{2}=\mathrm{RB}+\mathrm{E}_{2}+\mathrm{M}^{\text {Even }}$ with $E_{\text {Even }} \leftarrow B e r_{\tau}^{1 \times n}$ and $E_{2} \leftarrow B e r_{\tau}^{n \times n}$.

And second is Odd (), returns to ciphertext $\mathrm{C}=\left(\mathrm{C}_{1}\right.$ and $\left.\mathrm{C}_{\text {Odd }}\right)$ where the computations are $\mathrm{C}_{1}=\mathrm{RB}+\mathrm{E}_{1}+\mathrm{M}^{\text {Odd }}$ and $\mathrm{C}_{\text {Odd }}=\mathrm{RA}+\mathrm{E}_{\text {Odd }}$ with $E_{\text {Odd }} \leftarrow B e r_{\tau}^{n}$ and $E_{1} \leftarrow B e r_{\tau}^{n \times n}$.

3) kO-DLPN of kO_Dec( $s k, c)$, returns $\left(C_{1} \times\right.$ $\left.C_{\text {Even }}\right) \times S+\left(C_{2} \times C_{\text {odd }}\right)$.

\section{APpliCATION IS A WeB MODEL}

Proposed scheme is applicable for secure data encryption and decryption to eliminate the noise words between the users. This scheme improves the security information transmission between multi-users utilizing web benefit as an admin middle person. It identifies the noise information in the users' message which is in encrypted unscrambled by user side and by admin encryption the noise words are recognized and identified.

Separate models are made - 1) A web-application (user and admin support) and 2) A web-server (server support). Webapplication program encrypt user message received by admin through noisy message elimination during the decryption scheme and makes a web server benefit with noise-free message. The web-server receives the data processed from web applications, warns and removes user message information utilizing both sides of encryption and decryption. After admin information approval, web server restores the user message by eliminating the noise words and results the blocked user authentication for noise words presence and providing the message without any noise through the web application, which is illustrated in the below figures (Fig. 1 and 2).

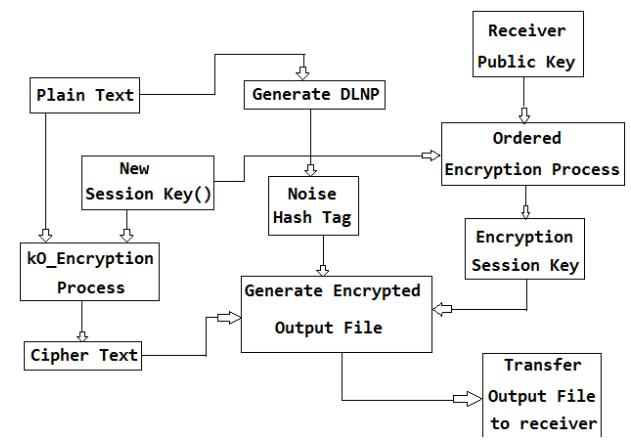

Fig. 1. Flowchart at the Sender Side.

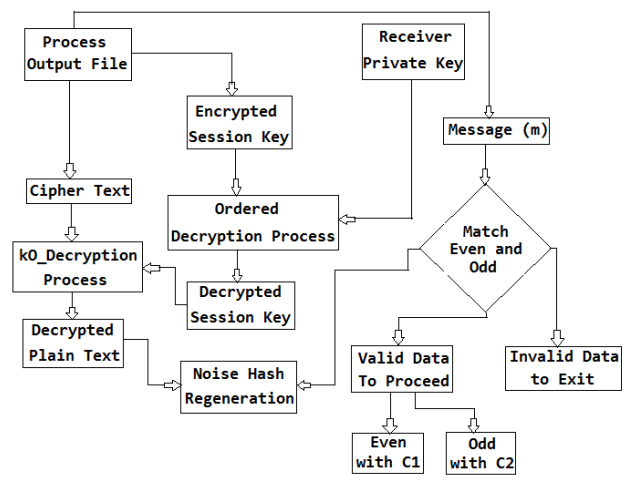

Fig. 2. Flow Chart at the Receiver Side. 


\section{RESUlTS AND DisCUSSIONS}

The proposed scheme uses Client layer through HTML and CSS, Business layer through java and jdbc and Persistence through web server. All these three are integrated on Apache NetBeans platform. Here 128-bit security is used with $n=29000$ through 3072-bit security levels. This work is reviewed by other methods through the following parameters.

\section{A. Key Generation Time}

Table I list the comparison between proposed scheme with Damgård schemes [18] and PPKE based LPN [29] in computational efficiency.

All the above are with respective to LPN, the multiplications and additions have made the computational time to reduce. Proposed work is similar to PPKELPN but proposed work increases slightly in public key and ciphertext in both the scenarios and decryption error can be neglected. From Table II, the key generation time of proposed work is better than the reviewed works. From the experiments it is proved that proposed work key generation time is higher than RSA, Table II shows these results.

\section{B. Encryption Time and Decryption Time}

Comparing the performance of proposed work with PPKELPN , RSA(not padding) and Damgård's scheme, the proposed work illustrates better than RSA, Table III shows these results.

Comparatively the method proposed scheme is better than the surveyed works.

TABLE. I. COMPARISON BETWEEN PROPOSED WORK AND DAMGÅRD SCHEMES AND PPKE BASED LPN

\begin{tabular}{|l|l|l|l|}
\hline Method & $\begin{array}{l}\text { Public key } \\
\text { size(bit) }\end{array}$ & $\begin{array}{l}\text { Ciphertext } \\
\text { size(bit) }\end{array}$ & $\begin{array}{l}\text { Encoding } \\
\text { error }\end{array}$ \\
\hline Damgård for bit=1 & $2 \mathrm{n}^{2}+2 \mathrm{n}$ & $\mathrm{n}+1$ & Yes \\
\hline PPKE based LPN for bit=1 & $2 \mathrm{n}^{2}$ & $2 \mathrm{n}$ & No \\
\hline Proposed for bit=1 & $\mathrm{n}^{2}$ & $\mathrm{~N}$ & No \\
\hline Damgård's for bit=multi & $4 \mathrm{n}^{2}$ & $2 \mathrm{n}$ & Yes \\
\hline PPKE based LPN for bit=multi & $2 \mathrm{n}^{2}$ & $2 \mathrm{n}^{2}$ & No \\
\hline Proposed for bit=multi & $2 \mathrm{n}^{2}$ & $\mathrm{n}^{2}+1$ & No \\
\hline
\end{tabular}

TABLE. II. COMPARISON BETWEEN PROPOSED WORK AND RSA, ERSA, HRSA IN KEY GENERATION TIME

\begin{tabular}{|l|l|}
\hline Security level (128 bits) & Key generation time (ms) \\
\hline RSA[24] & 0.127 \\
\hline ERSA [27] & 0.112 \\
\hline HRSA [28] & 0.241 \\
\hline Proposed Work & 0.352 \\
\hline
\end{tabular}

TABLE. III. COMPARISON BETWEEN PROPOSED WORK AND REVIEWED IN ENCRYPTION TIME AND DECRYPTION TIME

\begin{tabular}{|l|l|l|}
\hline Security level (128 bits) & Encryption time(ms) & Decryption time (ms) \\
\hline PPKE based LPN [29] & 102.10 & 0.258 \\
\hline Damgård's scheme [18] & 241.70 & 0.128 \\
\hline RSA(not padding) [12] & 0.060 & 2.890 \\
\hline Proposed Work & 99.85 & 0.119 \\
\hline
\end{tabular}

\section{CONCLUSIONS}

A cryptography scheme under public key through DLPN assumptions is an important research work, carrying many advantages comparatively. Due to decryption errors the existing systems are still having problems, which have to be corrected.

Through DLPN variant problem, a key-Ordered DLPN is proposed in this paper. There is a drastic change in the computing overhead of the proposed work compared to the PPKELPN, Damgård's scheme and RSA. Proposed work can withstand with the practical security like quantum attacks. A comparative result shows the proposed work gives high security.

\section{FUTURE SCOPE}

Further in future, through this work, design of public and private key cryptography to implement as a CCA level security, which can be made possible.

\section{REFERENCES}

[1] X. Sun, B. Li, X. Lu, and F. Fang, “CCA secure public key encryption scheme based on LWE without Gaussian sampling," in Proc. Int. Conf. Inf. Secur. Cryptol., vol. 9589, 2015, pp. 361378.

[2] Z. Liu, Y. Huang, J. Li, X. Cheng, and C. Shen, 'DivORAM: Towards a practical oblivious RAM with variable block size," Inf. Sci., vol. 447, pp. 111, Jun. 2018.

[3] C.-Z. Gao, Q. Cheng, P. He, W. Susilo, and J. Li, “'Privacy-preserving Naive Bayes classiers secure against the substitution-then-comparison attack," Inf. Sci., vol. 444, pp. 7288, May 2018.

[4] P. Li et al., "Multi-key privacy-preserving deep learning in cloud computing," Future Generat. Comput. Syst., vol. 74, pp. 7685, Sep. 2017.

[5] G. Liu, H. Li, and L. Yang, “'A topology preserving method of evolving contours based on sparsity constraint for object segmentation," IEEE Access, vol. 5, pp. 1997119982, 2017.

[6] N. Koblitz, A. Menezes, and S. Vanstone, “The state of elliptic curve cryptography," Des., Codes Cryptogr., vol. 19, nos. 23, pp. 173193, 2000.

[7] Z. Huang, S. Liu, X. Mao, K. Chen, and J. Li, ' Insight of the protection for data security under selective opening attacks," Inf. Sci., vols. 412413, pp. 223241, Oct. 2017.

[8] D. Boneh and A. Silverberg, “Applications of multilinear forms to cryptography," J. Contemp. Math., vol. 324, no. 1, pp. 7190, 2003.

[9] J.-S. Coron, T. Lepoint, and M. Tibouchi, 'Practical multilinear maps over the integers," in Advances in CryptologyCRYPTO (Lecture Notes in Computer Science), vol. 8042. Berlin, Germany: Springer, 2013, pp. 476493.

[10] Y. Hu and H. Jia, “Cryptanalysis of GGH map," Cryptol. ePrint Arch., Int. Assoc. Cryptol. Res., Tech. Rep., Feb. 2016. [Online]. Available: http://eprint.iacr.org/2015/301.pdf

[11] C. Gentry, C. Peikert, and V. Vaikuntanathan, "Trapdoors for hard lattices and new cryptographic constructions," in Proc. 40th Annu. ACM Symp.Theory Comput., 2008, pp. 197206

[12] J. Li, Y. Li, X. Chen, P. Lee, and W. Lou, “A hybrid cloud approach for secure authorized deduplication," IEEE Trans. Parallel Distrib. Syst., vol. 26, no. 5, pp. 12061216 May 2015.

[13] W. Chen, H. Lei, and K. Qi, "Lattice-based linearly homomorphic signatures in the standard model," Theor. Comput. Sci., vol. 634, pp. 4754, Jun. 2016.

[14] S. Garg, C. Gentry, S. Halevi, M. Raykova, A. Sahai, and B. Waters, "Candidate indistinguishability obfuscation and functional encryption for all circuits," in Proc. Annu. IEEE Symp. Found. Comput. Sci., Oct. 2013, pp. 4049. 
[15] A. Blum, A. Kalai, and H. Wasserman, "Noise-tolerant learning, the parity problem, and the statistical query model," J. ACM, vol. 50, no. 4, pp. 506519, 2003.

[16] P. Kirchner, "Improved generalized birthday attack," Cryptol. ePrint Arch., Int. Assoc. Cryptol. Res., Tech. Rep., Jun. 2016. [Online].Available: http://eprint.iacr.org/2011/377.pdf

[17] A. Juels and A. Stephen, “Authenticating pervasive devices with human protocols," in Proc. Annu. Int. Cryptol. Conf., vol. 3621, 2005, pp. 293308.

[18] J. Katz, J. S. Shin, and A. Smith, "Parallel and concurrent security of the HB and HBC protocols," J. Cryptol., vol. 23, no. 3 pp. 402421, 2010.

[19] I. Damgård and S. Park, "How practical is public-key encryption based on LPN and ring-LPN?" Cryptol. ePrint Arch., Int. Assoc. Cryptol. Res., Tech. Rep., Jun. 2016. [Online]. Available: http://eprint.iacr.org 12012/699.pdf

[20] W. Stallings, "Cryptography and network security: principles and practice", sixth edition, 2014, ISBN: 0-13-335469-5, pp. 9-60, 253285.

[21] R. Rivest, A. Shamir and L. Adleman, "A method for obtaining digital signatures and public key cryptosystems", Communications of the ACM vol. 21 (2), pp.120-126, 1978.

[22] R S Dhakar, A K Gupta and P Sharma, "Modified RSA encryption algorithm (MREA)”, 2nd ICACCT, IEEE, pp. 426-429, 2012.
[23] M.Thangavel, P. Varalakshmi, M. Murrali and K.Nithya, "An enhanced and secured RSA key generation scheme" Journal of Information Security and applications, Elsevier, vol 20, pp.3-10, 2015.

[24] F.Kong, J. Yu and L. Wu, "Security analysis of an RSA key generation algorithm with a large private key", Springer- Verlag Berlin Heidelberg, PP-95-101, 2011.

[25] L. H. Encinas, J. M. Masqu'e and A. Q. Dios, "An algorithm to obtain a RSA modulus with a large private key", Cryptology ePrint Archive: Report 2003/045.

[26] B.R. Ambedkar, A. Gupta,P. Gautam and S.S.Bedi, "An Efficient Method to Factorize the RSA Public Key Encryption." Communication Systems and Network Technologies (CSNT), 2011 International Conference on

[27] R. Minni, K. Sultania and S.Mishra, "An algorithm to enhance security in RSA", 4th ICCCNT, IEEE, pp.1-4, 2013.

[28] Jayraj Gondaliya at all.,Hybrid Security RSA Algorithm in Application of Web Service,2018 1st International Conference on Data Intelligence and Security, (O2018 IEEE, pp-149-152.

[29] Zhimin Yu at all.,A Practical Public Key Encryption Scheme Based on Learning Parity With Noise, Special Section On Information Security Solutions For Telemedicine Applications, IEEE Access, VOLUME 6, 2018, pp-31918-31923. 